\title{
Measurement of the tau branching fractions into leptons
}

\section{L3 Collaboration}

M. Acciarri ${ }^{\mathrm{y}}$, P. Achard ${ }^{\mathrm{s}}, \mathrm{O}$. Adriani ${ }^{\mathrm{p}}, \mathrm{M}$. Aguilar-Benitez ${ }^{\mathrm{x}}, \mathrm{J}$ Alcaraz $^{\mathrm{x}}$, G. Alemanni ${ }^{u}$, J. Allaby ${ }^{\mathrm{q}}$, A. Aloisio ${ }^{\text {aa }}$, M.G. Alviggi ${ }^{\text {aa }}$, G. Ambrosi ${ }^{\mathrm{s}}, \mathrm{H}$. Anderhub ${ }^{\text {au }}$, V.P. Andreev f,af , T. Angelescu ${ }^{1}$, F. Anselmo ${ }^{i}$, A. Arefiev ${ }^{z}$, T. Azemoon ${ }^{\text {c }}$, T. Aziz ${ }^{j}$, P. Bagnaia ${ }^{\text {ak }}$, A. Bajo ${ }^{\mathrm{x}}$, L. Baksay ${ }^{\text {ap }}$, A. Balandras ${ }^{\mathrm{d}}$, S.V. Baldew ${ }^{\mathrm{b}}$, S. Banerjee $^{\mathrm{j}}$, Sw. Banerjee ${ }^{\mathrm{d}}$, A. Barczyk ${ }^{\text {au, as }}$, R. Barillère ${ }^{\mathrm{q}}$, P. Bartalini ${ }^{u}$, M. Basile ${ }^{\mathrm{i}}, \mathrm{N}$. Batalova ${ }^{\text {ar }}$,

R. Battiston ${ }^{\text {ae }}$, A. Bay ${ }^{\mathrm{u}}$, F. Becattini ${ }^{\mathrm{p}}, \mathrm{U}$. Becker ${ }^{\mathrm{n}}$, F. Behner ${ }^{\mathrm{au}}$, L. Bellucci $^{\mathrm{p}}$, R. Berbeco ${ }^{c}$, J. Berdugo ${ }^{x}$, P. Berges ${ }^{n}$, B. Bertucci ${ }^{\text {ae }}$, B.L. Betev ${ }^{\text {au }}$, S. Bhattacharya ${ }^{j}$, M. Biasini ${ }^{\text {ae }}$, A. Biland ${ }^{\text {au }}$, J.J. Blaising ${ }^{\text {d, S.C. Blyth }}{ }^{\text {ag }}$, G.J. Bobbink ${ }^{\text {b }}$, A. Böhm ${ }^{\text {a }}$, L. Boldizsar ${ }^{\mathrm{m}}$, B. Borgia ${ }^{\text {ak }}$, D. Bourilkov ${ }^{\mathrm{au}}$, M. Bourquin ${ }^{\mathrm{s}}$, S. Braccini ${ }^{\mathrm{s}}$, J.G. Branson ${ }^{\text {am }}$, F. Brochu ${ }^{\mathrm{d}}$, A. Buffini ${ }^{\mathrm{p}}$, A. Buijs ${ }^{\mathrm{aq}}$, J.D. Burger ${ }^{\mathrm{n}}$, W.J. Burger ${ }^{\text {ae }}$, X.D. Cai ${ }^{\mathrm{n}}$, M. Capell ${ }^{\mathrm{n}}$, G. Cara Romeo ${ }^{\mathrm{i}}$, G. Carlino ${ }^{\text {aa }}$, A.M. Cartacci ${ }^{\mathrm{p}}$, J. Casaus $^{\mathrm{x}}$, G. Castellini $^{\mathrm{p}}$, F. Cavallari ${ }^{\text {ak }}$, N. Cavallo ${ }^{\text {ah }}$, C. Cecchi ${ }^{\text {ae }}$, M. Cerrada ${ }^{\mathrm{x}}$, F. Cesaroni $^{\mathrm{v}}$, M. Chamizo ${ }^{\text {s }}$, Y.H. Chang ${ }^{\text {aw }}$, U.K. Chaturvedi ${ }^{r}$, M. Chemarin ${ }^{w}$, A. Chen ${ }^{\text {aw }}$, G. Chen ${ }^{g}$, G.M. Chen ${ }^{\mathrm{g}}$, H.F. Chen ${ }^{\mathrm{t}}$, H.S. Chen ${ }^{\mathrm{g}}$, G. Chiefari ${ }^{\mathrm{a} a}$, L. Cifarelli ${ }^{\text {al }}$, F. Cindolo ${ }^{\mathrm{i}}$, C. Civinini $^{\mathrm{p}}$, I. Clare ${ }^{\mathrm{n}}$, R. Clare ${ }^{\mathrm{aj}}$, G. Coignet $^{\mathrm{d}}$, A.P. Colijn ${ }^{\mathrm{b}}$, N. Colino ${ }^{\mathrm{x}}$, S. Costantini ${ }^{\mathrm{e}}$, F. Cotorobai ${ }^{1}$, B. de la Cruz ${ }^{\mathrm{x}}$, A. Csilling ${ }^{\mathrm{m}}$, S. Cucciarelli ${ }^{\text {ae }}$, T.S. Dai ${ }^{\mathrm{n}}$, J.A. van Dalen ${ }^{\text {ac }}$, R. D’Alessandro ${ }^{p}$, R. de Asmundis ${ }^{\text {aa }}$, P. Déglon ${ }^{\mathrm{s}}$, A. Degré ${ }^{\mathrm{d}}$, K. Deiters ${ }^{\text {as }}$, D. della Volpe ${ }^{\text {aa }}$, E. Delmeire ${ }^{\text {s }}$, P. Denes ${ }^{\text {ai }}$, F. DeNotaristefani ${ }^{\text {ak }}$, A. De Salvo ${ }^{\text {au }}$, M. Diemoz ak ${ }^{\text {a }}$ M. Dierckxsens ${ }^{\text {b }}$, D. van Dierendonck ${ }^{\mathrm{b}}$, C. Dionisi ak, M. Dittmar ${ }^{\text {au }}$, A. Dominguez ${ }^{\text {am }}$, A. Doria ${ }^{\text {aa }}$, M.T. Dova ${ }^{\text {r, } 1}$, D. Duchesneau ${ }^{\mathrm{d}}$, D. Dufournaud ${ }^{\text {d }}$, P. Duinker ${ }^{\text {b }}$, H. El Mamouni ${ }^{\text {w }}$, A. Engler ${ }^{\text {ag }}$, F.J. Eppling ${ }^{\text {n, }}$, F.C. Erné ${ }^{\mathrm{b}}$, A. Ewers ${ }^{\mathrm{a}}$, P. Extermann ${ }^{\mathrm{s}}$, M. Fabre ${ }^{\text {as }}$, M.A. Falagan ${ }^{\mathrm{x}}$, S. Falciano ${ }^{\text {ak, }}{ }^{\text {, }}$, A. Favara ${ }^{\text {, }}$ J. Fay ${ }^{\text {w }}$, O. Fedin ${ }^{\text {af }}$, M. Felcini ${ }^{\text {au }}$, T. Ferguson ${ }^{\text {ag }}$, H. Fesefeldt ${ }^{\text {a }}$, E. Fiandrini ${ }^{\text {ae }}$, J.H. Field ${ }^{\text {s }}$, F. Filthaut ${ }^{\mathrm{q}}$, P.H. Fisher ${ }^{\mathrm{n}}$, I. Fisk ${ }^{\text {am }}$, G. Forconi ${ }^{\mathrm{n}}$, K. Freudenreich ${ }^{\text {au }}$, C. Furetta ${ }^{y}$, Yu. Galaktionov ${ }^{z, n}$, S.N. Ganguli ${ }^{j}$, P. Garcia-Abia ${ }^{\mathrm{e}}$, M. Gataullin ${ }^{\text {ad }}$, S.S. Gau ${ }^{k}$, S. Gentile ${ }^{\mathrm{ak}, \mathrm{q}}$, N. Gheordanescu ${ }^{1}$, S. Giagu ${ }^{\text {ak }}$, Z.F. Gong ${ }^{\mathrm{t}}$, G. Grenier ${ }^{\mathrm{w}}$, O. Grimm ${ }^{\text {au }}$, M.W. Gruenewald ${ }^{\mathrm{h}}, \mathrm{M}$ Guida $^{\mathrm{al}}$, R. van Gulik ${ }^{\mathrm{b}}$, V.K. Gupta ${ }^{\text {ai }}$, A. Gurtu ${ }^{\text {j }}$, L.J. Gutay ${ }^{\text {ar }}$, D. Haas ${ }^{\text {e }}$, A. Hasan ${ }^{\text {ab }}$, D. Hatzifotiadou ${ }^{\mathrm{i}}$, T. Hebbeker ${ }^{\text {h}}$, A. Hervé ${ }^{\mathrm{q}}$, P. Hidas ${ }^{\mathrm{m}}$, J. Hirschfelder ${ }^{\mathrm{ag}}$, H. Hofer ${ }^{\text {au }}$, G. Holzner ${ }^{\text {au }}$, H. Hoorani ${ }^{\text {ag }}$, S.R. Hou ${ }^{\text {aw }}$, Y. Hu ${ }^{\text {ac }}$, I. Iashvili ${ }^{\text {at }}$, B.N. Jin ${ }^{\mathrm{g}}$, L.W. Jones ${ }^{\mathrm{c}}$, P. de Jong ${ }^{\mathrm{b}}$, 
I. Josa-Mutuberría ${ }^{\mathrm{x}}$, R.A. Khan ${ }^{\mathrm{r}}$, D. Käfer ${ }^{\mathrm{a}}$, M. Kaur ${ }^{\mathrm{r}, 2}$, M.N. Kienzle-Focacci ${ }^{\text {s, }}$ D. Kim ${ }^{\text {ak }}$, J.K. Kim ${ }^{\text {ao }}$, J. Kirkby ${ }^{\mathrm{q}}$, D. Kiss ${ }^{\mathrm{m}}$, W. Kittel ${ }^{\mathrm{ac}}$, A. Klimentov ${ }^{\mathrm{n}, \mathrm{z}}$, A.C. König ${ }^{\text {ac }}$, M. Kopal ${ }^{\text {ar }}$, A. Kopp ${ }^{\text {at }}$, V. Koutsenko ${ }^{\text {n,z }}$, M. Kräber ${ }^{\text {au }}$, R.W. Kraemer ${ }^{\text {ag }}$, W. Krenz ${ }^{\text {a }}$, A. Krüger ${ }^{\text {at }}$, A. Kunin ${ }^{\mathrm{n}, \mathrm{z}}$, P. Lacentre ${ }^{\text {at, } 3}$, P. Ladron de Guevara ${ }^{\mathrm{x}}$, I. Laktineh ${ }^{\mathrm{w}}$, G. Landi ${ }^{\mathrm{p}}$, M. Lebeau ${ }^{\mathrm{q}}$, A. Lebedev ${ }^{\mathrm{n}}$, P. Lebrun ${ }^{\mathrm{w}}$, P. Lecomte ${ }^{\text {au }}$, P. Lecoq ${ }^{q}$, P. Le Coultre ${ }^{\text {au }}$, H.J. Lee ${ }^{\text {h}}$, J.M. Le Goff ${ }^{\text {, }}$, R. Leiste ${ }^{\text {at }}$, P. Levtchenko ${ }^{\text {af }}$, C. Li $^{t}$, S. Likhoded ${ }^{\text {at }}$, C.H. Lin ${ }^{\text {aw }}$, W.T. Lin ${ }^{\text {aw }}$, F.L. Linde ${ }^{\text {b }}$, L. Lista ${ }^{\text {aa }}$, Z.A. Liu ${ }^{\text {, }}$, W. Lohmann ${ }^{\text {at }}$, E. Longo ${ }^{\text {ak }}$, Y.S. Lu ${ }^{\mathrm{g}}$, K. Lübelsmeyer ${ }^{\mathrm{a}}$, C. Luci ${ }^{\mathrm{q}, \mathrm{ak}}$, D. Luckey ${ }^{\mathrm{n}}$, L. Lugnier ${ }^{\mathrm{w}}$, L. Luminari ${ }^{\text {ak }}$, W. Lustermann ${ }^{\text {au }}$, W.G. Ma ${ }^{\mathrm{t}}$, M. Maity ${ }^{\mathrm{j}}$, L. Malgeri ${ }^{\mathrm{s}}$, A. Malinin ${ }^{\text {q }}$, C. Maña ${ }^{\mathrm{x}}$, D. Mangeol ${ }^{\text {ac }}$, J. Mans ${ }^{\text {ai }}$, G. Marian ${ }^{\circ}$, J.P. Martin ${ }^{\text {w }}$, F. Marzano ${ }^{\text {ak }}$, K. Mazumdar ${ }^{\mathrm{j}}$, R.R. McNeil ${ }^{\mathrm{f}}$, S. Mele ${ }^{\mathrm{q}}$, L. Merola ${ }^{\text {aa }}$, M. Meschini ${ }^{\mathrm{p}}$, W.J. Metzger ac , M. von der Mey ${ }^{a}$, A. Mihul ${ }^{1}$, H. Milcent ${ }^{\mathrm{q}}$, G. Mirabelli ${ }^{\text {ak }}$, J. Mnich ${ }^{\text {a }}$, G.B. Mohanty ${ }^{\mathrm{j}}$, R. Moore ${ }^{\mathrm{c}}$, T. Moulik ${ }^{\mathrm{j}}$, G.S. Muanza ${ }^{\mathrm{w}}$, A.J.M. Muijs ${ }^{\mathrm{b}}$, B. Musicar ${ }^{\mathrm{am}}$, M. Musy ${ }^{\text {ak }}$, M. Napolitano a, ${ }^{\text {aa }}$ F. Nessi-Tedaldi ${ }^{\text {au }}$, H. Newman ${ }^{\text {ad }}$, T. Niessen ${ }^{\text {a }}$, A. Nisati ${ }^{\text {ak }}, \mathrm{H}$. Nowak $^{\text {at }}$, R. Ofierzynski ${ }^{\text {au }}$, G. Organtini ${ }^{\text {ak }}$, A. Oulianov ${ }^{\mathrm{z}}$, C. Palomares ${ }^{\mathrm{x}}$, D. Pandoulas ${ }^{\mathrm{a}}$, S. Paoletti ${ }^{\text {ak, }}$, P. Paolucci ${ }^{\text {aa }}$, R. Paramatti ${ }^{\text {ak }}$, H.K. Park ${ }^{\text {ag }}$, I.H. Park ${ }^{\text {ao }}$, G. Passaleva ${ }^{\text {q }}$, S. Patricelli ${ }^{\text {aa }}$, T. Paul ${ }^{\text {k }}$, M. Pauluzzi ${ }^{\text {ae }}$, C. Paus ${ }^{\mathrm{q}}$, F. Pauss ${ }^{\mathrm{au}}$, M. Pedace ${ }^{\mathrm{ak}}$, S. Pensotti ${ }^{\mathrm{y}}$, D. Perret-Gallix ${ }^{\mathrm{d}}$, B. Petersen ${ }^{\text {ac }}$,

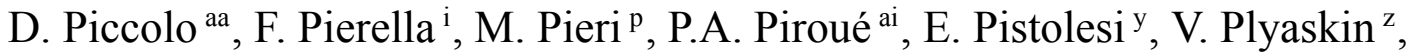
M. Pohl ${ }^{\text {s }}$, V. Pojidaev ${ }^{z, p}$, H. Postema ${ }^{\text {n }}$, J. Pothier ${ }^{\mathrm{q}}$, D.O. Prokofiev ${ }^{\text {ar }}$, D. Prokofiev ${ }^{\text {af }}$, J. Quartieri ${ }^{\text {al }}$, G. Rahal-Callot ${ }^{\text {au, }}$, M.A. Rahaman ${ }^{j}$, P. Raics ${ }^{\circ}$, N. Raja ${ }^{j}$, R. Ramelli ${ }^{\text {au }}$, P.G. Rancoita ${ }^{\mathrm{y}}$, R. Ranieri ${ }^{\mathrm{p}}$, A. Raspereza ${ }^{\text {at }}, \mathrm{G}$. Raven ${ }^{\mathrm{am}}{ }$, P. Razis ${ }^{\mathrm{ab}}$, D. Ren ${ }^{\mathrm{au}}$, M. Rescigno ${ }^{\text {ak }}$, S. Reucroft ${ }^{k}$, S. Riemann ${ }^{\text {at }}$, K. Riles ${ }^{\text {c }}$, J. Rodin ${ }^{\text {ap }}$, B.P. Roe ${ }^{\text {c }}$, L. Romero ${ }^{\mathrm{x}}$, A. Rosca ${ }^{\mathrm{h}}, \mathrm{S}$. Rosier-Lees ${ }^{\mathrm{d}}$, S. Roth ${ }^{\mathrm{a}}$, C. Rosenbleck $^{\mathrm{a}}$, B. Roux ${ }^{\text {ac }}$, J.A. Rubio ${ }^{q}$, G. Ruggiero ${ }^{p}$, H. Rykaczewski ${ }^{\text {au }}$, S. Saremi ${ }^{\text {f }}$, S. Sarkar ${ }^{\text {ak }}$, J. Salicio ${ }^{q}$,

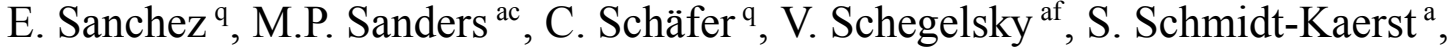
D. Schmitz ${ }^{\text {a }}$, H. Schopper ${ }^{\text {av }}$, D.J. Schotanus ${ }^{\text {ac }}$, G. Schwering ${ }^{\text {a }}$, C. Sciacca ${ }^{\text {aa }}$, A. Seganti ${ }^{\text {i }}$ L. Servoli ${ }^{\text {ae }}$, S. Shevchenko ${ }^{\text {ad }}$, N. Shivarov an, V. Shoutko ${ }^{\text {z }}$, E. Shumilov ${ }^{\text {, }}$, A. Shvorob ${ }^{\text {ad }}$, T. Siedenburg ${ }^{\text {a }}$, D. Son ${ }^{\text {ao }}$, B. Smith ${ }^{\text {ag }}$, P. Spillantini ${ }^{p}$, M. Steuer ${ }^{n}$, D.P. Stickland ${ }^{\text {ai }}$, A. Stone ${ }^{\text {f }}$, B. Stoyanov ${ }^{\text {an }}$, A. Straessner ${ }^{\mathrm{q}}$, K. Sudhakar ${ }^{\mathrm{j}}$, G. Sultanov ${ }^{\mathrm{r}}$, L.Z. Sun ${ }^{\text {t }}$, S. Sushkov ${ }^{\text {h }}$, H. Suter ${ }^{\text {au }}$, J.D. Swain ${ }^{\text {r }}$, Z. Szillasi ${ }^{\text {ap, } 6}$, T. Sztaricskai ${ }^{\text {ap, }}{ }^{\text {, }}$, X.W. Tang ${ }^{\mathrm{g}}$, L. Tauscher ${ }^{\mathrm{e}}$, L. Taylor ${ }^{\mathrm{k}}$, B. Tellili ${ }^{\mathrm{w}}$, D. Teyssier ${ }^{\mathrm{w}}$, C. Timmermans ${ }^{\mathrm{ac}}$, Samuel C.C. Ting ${ }^{n}$, S.M. Ting ${ }^{n}$, S.C. Tonwar ${ }^{j}$, J. Tóth ${ }^{\mathrm{m}}$, C. Tully ${ }^{\mathrm{q}}$, K.L. Tung ${ }^{\mathrm{g}}$, Y. Uchida ${ }^{\mathrm{n}}$, J. Ulbricht ${ }^{\mathrm{au}}$, E. Valente ${ }^{\mathrm{ak}}$, G. Vesztergombi $^{\mathrm{m}}$, I. Vetlitsky ${ }^{\mathrm{z}}$, D. Vicinanza ${ }^{\text {al }}$, G. Viertel ${ }^{\text {au }}$, S. Villa ${ }^{\text {aj }}$, M. Vivargent ${ }^{\mathrm{d}}$, S. Vlachos ${ }^{\mathrm{e}}, \mathrm{I}_{\text {. Vodopianov }}{ }^{\text {af }}$, H. Vogel ${ }^{\text {ag }}, H$. Vogt ${ }^{\text {at }}$, I. Vorobiev ${ }^{\text {ag }}$, A.A. Vorobyov ${ }^{\text {af }}$, A. Vorvolakos ${ }^{\text {ab }}$, M. Wadhwa ${ }^{\mathrm{e}}$, W. Wallraff ${ }^{\mathrm{a}}$, M. Wang ${ }^{\mathrm{n}}$, X.L. Wang ${ }^{\mathrm{t}}$, Z.M. Wang ${ }^{\mathrm{t}}$, A. Weber ${ }^{\mathrm{a}}$, M. Weber ${ }^{\mathrm{a}}$, P. Wienemann ${ }^{\mathrm{a}}$, H. Wilkens ${ }^{\mathrm{ac}}$, S.X. Wu ${ }^{\mathrm{n}}$, S. Wynhoff ${ }^{\mathrm{q}}$, L. Xia ${ }^{\text {ad }}$, Z.Z. Xu ${ }^{\mathrm{t}}$, 
J. Yamamoto $^{\text {c }}$, B.Z. Yang ${ }^{\mathrm{t}}$, C.G. Yang ${ }^{\mathrm{g}}$, H.J. Yang ${ }^{\mathrm{g}}$, M. Yang ${ }^{\mathrm{g}}$, J.B. Ye ${ }^{\mathrm{t}}$, S.C. Yeh ${ }^{\mathrm{ax}}$, An. Zalite ${ }^{\text {af }}$, Yu. Zalite ${ }^{\text {af }}$, Z.P. Zhang ${ }^{\mathrm{t}}$, G.Y. Zhu ${ }^{\mathrm{g}}$, R.Y. Zhu ${ }^{\text {ad }}$, A. Zichichi ${ }^{\mathrm{i}, \mathrm{q}, \mathrm{r}}$, F. Ziegler ${ }^{\text {at }}$, G. Zilizi ${ }^{\text {ap, }}$, B. Zimmermann ${ }^{\text {au }}$, M. Zöller ${ }^{\text {a }}$

a I. Physikalisches Institut, RWTH, D-52056 Aachen, Germany, III. Physikalisches Institut, RWTH, D-52056 Aachen, Germany 4

b National Institute for High Energy Physics, NIKHEF, and University of Amsterdam, NL-1009 DB Amsterdam, The Netherlands

${ }^{c}$ University of Michigan, Ann Arbor, MI 48109, USA

d Laboratoire d'Annecy-le-Vieux de Physique des Particules, LAPP, IN2P3-CNRS, BP 110, F-74941 Annecy-le-Vieux CEDEX, France

e Institute of Physics, University of Basel, CH-4056 Basel, Switzerland

${ }^{\mathrm{f}}$ Louisiana State University, Baton Rouge, LA 70803, USA

$\mathrm{g}$ Institute of High Energy Physics, IHEP, 100039 Beijing, PR China ${ }^{8}$

h Humboldt University, D-10099 Berlin, Germany 4

${ }^{\mathrm{i}}$ University of Bologna and INFN-Sezione di Bologna, I-40126 Bologna, Italy

$\mathrm{j}$ Tata Institute of Fundamental Research, Bombay 400 005, India

${ }^{\mathrm{k}}$ Northeastern University, Boston, MA 02115, USA

${ }^{1}$ Institute of Atomic Physics and University of Bucharest, R-76900 Bucharest, Romania

${ }^{\mathrm{m}}$ Central Research Institute for Physics of the Hungarian Academy of Sciences, H-1525 Budapest 114, Hungary ${ }^{5}$

${ }^{\mathrm{n}}$ Massachusetts Institute of Technology, Cambridge, MA 02139, USA

${ }^{\circ}$ KLTE-ATOMKI, H-4010 Debrecen, Hungary 6

$\mathrm{p}$ INFN Sezione di Firenze and University of Florence, I-50125 Florence, Italy

${ }^{\mathrm{q}}$ European Laboratory for Particle Physics, CERN, CH-1211 Geneva 23, Switzerland

${ }^{\mathrm{r}}$ World Laboratory, FBLJA Project, CH-1211 Geneva 23, Switzerland

${ }^{\mathrm{s}}$ University of Geneva, CH-1211 Geneva 4, Switzerland

${ }^{\mathrm{t}}$ Chinese University of Science and Technology, USTC, Hefei, Anhui 230 029, PR China 8

u University of Lausanne, CH-1015 Lausanne, Switzerland

${ }^{v}$ INFN-Sezione di Lecce and Università Degli Studi di Lecce, I-73100 Lecce, Italy

w Institut de Physique Nucléaire de Lyon, IN2P3-CNRS, Université Claude Bernard, F-69622 Villeurbanne, France

${ }^{\mathrm{x}}$ Centro de Investigaciones Energéticas, Medioambientales y Tecnologícas, CIEMAT, E-28040 Madrid, Spain ${ }^{7}$

y INFN-Sezione di Milano, I-20133 Milan, Italy

${ }^{\mathrm{z}}$ Institute of Theoretical and Experimental Physics, ITEP, Moscow, Russia

aa INFN-Sezione di Napoli and University of Naples, I-80125 Naples, Italy

${ }^{\mathrm{ab}}$ Department of Natural Sciences, University of Cyprus, Nicosia, Cyprus

ac University of Nijmegen and NIKHEF, NL-6525 ED Nijmegen, The Netherlands

ad California Institute of Technology, Pasadena, CA 91125, USA

ae INFN-Sezione di Perugia and Università Degli Studi di Perugia, I-06100 Perugia, Italy

${ }^{\text {af }}$ Nuclear Physics Institute, St. Petersburg, Russia

ag Carnegie Mellon University, Pittsburgh, PA 15213, USA

ah INFN-Sezione di Napoli and University of Potenza, I-85100 Potenza, Italy

ai Princeton University, Princeton, NJ 08544, USA

aj University of California, Riverside, CA 92521, USA

ak INFN-Sezione di Roma and University of Rome "La Sapienza", I-00185 Rome, Italy

al University and INFN, Salerno, I-84100 Salerno, Italy

am University of California, San Diego, CA 92093, USA

an Bulgarian Academy of Sciences, Central Lab. of Mechatronics and Instrumentation, BU-1113 Sofia, Bulgaria

${ }^{\text {ao }}$ Laboratory of High Energy Physics, Kyungpook National University, $702-701$ Taegu, South Korea

ap University of Alabama, Tuscaloosa, AL 35486, USA

aq Utrecht University and NIKHEF, NL-3584 CB Utrecht, The Netherlands

${ }^{\text {ar }}$ Purdue University, West Lafayette, IN 47907, USA

as Paul Scherrer Institut, PSI, CH-5232 Villigen, Switzerland

at DESY, D-15738 Zeuthen, Germany

${ }^{\text {au }}$ Eidgenössische Technische Hochschule, ETH Zürich, CH-8093 Zürich, Switzerland

av University of Hamburg, D-22761 Hamburg, Germany

${ }^{\text {aw }}$ National Central University, Chung-Li, Taiwan, ROC

ax Department of Physics, National Tsing Hua University, Taiwan, ROC

Received 29 January 2001; accepted 13 February 2001

Editor: K. Winter 


\begin{abstract}
Using data collected with the L3 detector near the $\mathrm{Z}$ resonance, corresponding to an integrated luminosity of $150 \mathrm{pb}^{-1}$, the branching fractions of the tau lepton into electron and muon are measured to be $\mathcal{B}\left(\tau \rightarrow \mathrm{e} \overline{\mathrm{e}}_{\mathrm{e}} \nu_{\tau}\right)=(17.806 \pm 0.104$ (stat.) \pm 0.076 (syst.) $) \%, \mathcal{B}\left(\tau \rightarrow \mu \bar{v}_{\mu} \nu_{\tau}\right)=(17.342 \pm 0.110$ (stat.) \pm 0.067 (syst. $\left.)\right) \%$. From these results the ratio of the charged current coupling constants of the muon and the electron is determined to be $g_{\mu} / g_{\mathrm{e}}=1.0007 \pm 0.0051$. Assuming electron-muon universality, the Fermi constant is measured in tau lepton decays as $G_{F}=(1.1616 \pm 0.0058) \times 10^{-5} \mathrm{GeV}^{-2}$. Furthermore, the coupling constant of the strong interaction at the tau mass scale is obtained as $\alpha_{S}\left(m_{\tau}^{2}\right)=0.322 \pm 0.009$ (exp.) \pm 0.015 (theory). (C) 2001 Elsevier Science B.V. All rights reserved.
\end{abstract}

\section{Introduction}

In the Standard Model of electroweak interactions [1], the couplings of the leptons to the gauge bosons are assumed to be independent of the lepton generation. Measurements of the leptonic branching fractions $\mathcal{B}\left(\tau \rightarrow \ell \bar{\nu}_{\ell} \nu_{\tau}\right)$ and of the lifetime $\tau_{\tau}$ of the tau lepton provide a test of this universality hypothesis for the charged current. The leptonic width of the tau lepton [2],

$$
\begin{aligned}
\Gamma & \left(\tau \rightarrow \ell \bar{v}_{\ell} \nu_{\tau}\right) \\
& \equiv \frac{\mathcal{B}\left(\tau \rightarrow \ell \bar{v}_{\ell} \nu_{\tau}\right)}{\tau_{\tau}} \\
& =\frac{g_{\tau}^{2} g_{\ell}^{2}}{m_{\mathrm{W}}^{4}} \frac{m_{\tau}^{5}}{96(4 \pi)^{3}}\left(1+\epsilon_{P}\right)\left(1+\epsilon_{\mathrm{QED}}\right)\left(1+\epsilon_{q^{2}}\right),
\end{aligned}
$$

where $\ell=\mathrm{e}, \mu$, depends on the coupling constants of the tau lepton and the lepton $\ell$ to the W boson, $g_{\tau}$ and $g_{\ell}$, respectively. Here $m_{\tau}$ and $m_{\mathrm{W}}$ are the masses

\footnotetext{
1 Also supported by CONICET and Universidad Nacional de La Plata, CC 67, 1900 La Plata, Argentina.

2 Also supported by Panjab University, Chandigarh-160014, India.

3 Also supported by Deutscher akademischer Austauschdienst.

4 Supported by the German Bundesministerium für Bildung, Wissenschaft, Forschung und Technologie.

5 Supported by the Hungarian OTKA fund under contract numbers T019181, F023259 and T024011.

6 Also supported by the Hungarian OTKA fund under contract numbers T22238 and T026178.

7 Supported also by the Comisión Interministerial de Ciencia y Tecnología.

${ }^{8}$ Supported by the National Natural Science Foundation of China.
}

of the tau lepton and the $\mathrm{W}$ boson. The quantities $\epsilon_{P}, \epsilon_{\mathrm{QED}}$ and $\epsilon_{q^{2}}$ are small corrections resulting from phase-space, QED corrections [3] and the W propagator $[4,5]$, respectively.

The comparison of the tau branching fractions into electron and muon gives a direct measurement of the ratio $g_{\mu} / g_{\mathrm{e}}$. Moreover, tau decays into hadrons are sensitive to the strong coupling constant $\alpha_{s}$ at the tau mass scale. The ratio of the hadronic to the electronic width, $R_{\tau}$, can be expressed in terms of the leptonic branching fractions:

$$
\begin{aligned}
R_{\tau} & =\frac{\mathcal{B}\left(\tau \rightarrow h \nu_{\tau}\right)}{\mathcal{B}\left(\tau \rightarrow \mathrm{e} \bar{v}_{\mathrm{e}} \nu_{\tau}\right)} \\
& =\frac{1-\mathcal{B}\left(\tau \rightarrow \mathrm{e} \bar{\nu}_{\mathrm{e}} \nu_{\tau}\right)-\mathcal{B}\left(\tau \rightarrow \mu \bar{v}_{\mu} \nu_{\tau}\right)}{\mathcal{B}\left(\tau \rightarrow \mathrm{e} \bar{v}_{\mathrm{e}} \nu_{\tau}\right)},
\end{aligned}
$$

where $\mathcal{B}\left(\tau \rightarrow h v_{\tau}\right)$ is the branching fraction of the $\tau$ into hadrons. $R_{\tau}$ is calculated in perturbative QCD $[6,7]$ as:

$$
\begin{aligned}
R_{\tau}= & 3\left(\left|V_{\mathrm{ud}}\right|^{2}+\left|V_{\mathrm{us}}\right|^{2}\right) S_{\mathrm{EW}} \\
& \times\left(1+\frac{\alpha_{s}}{\pi}+5.2023\left(\frac{\alpha_{s}}{\pi}\right)^{2}+26.366\left(\frac{\alpha_{s}}{\pi}\right)^{3}\right. \\
& \left.+\left(78+d_{3}\right)\left(\frac{\alpha_{s}}{\pi}\right)^{4}+\delta_{\mathrm{NP}}\right)
\end{aligned}
$$

where $V_{\mathrm{ud}}$ and $V_{\mathrm{us}}$ are elements of the CabibboKobayashi-Maskawa (CKM) quark mixing matrix [8]. The quantities $S_{\mathrm{EW}}$ [9] and $\delta_{\mathrm{NP}}$ [10] describe short range electroweak radiative corrections and nonperturbative QCD contributions, respectively. The quantity $d_{3}$ is estimated to be 27.5 [7], to which we assign a $100 \%$ uncertainty.

This Letter presents a measurement of the tau branching fractions into electron and muon with the 
L3 detector [11] at LEP using data taken near the Z pole. The results supersede our previously published ones [12]. Results from other experiments are reported elsewhere [13].

\section{Data sample and Monte Carlo simulation}

The data were collected from 1991 to $1995^{9}$ at centre-of-mass energies around the $\mathrm{Z}$ mass and correspond to an integrated luminosity of about $150 \mathrm{pb}^{-1}$.

For efficiency and background estimates, Monte Carlo events are generated using the programs KORALZ [14] for $\mathrm{e}^{+} \mathrm{e}^{-} \rightarrow \mu^{+} \mu^{-}(\gamma)$ and $\mathrm{e}^{+} \mathrm{e}^{-} \rightarrow$ $\tau^{+} \tau^{-}(\gamma)$, BHAGENE [15] for $\mathrm{e}^{+} \mathrm{e}^{-} \rightarrow \mathrm{e}^{+} \mathrm{e}^{-}(\gamma)$, DIAG36 [16] for $\mathrm{e}^{+} \mathrm{e}^{-} \rightarrow \mathrm{e}^{+} \mathrm{e}^{-} \overline{f f}$, where ff is $\mathrm{e}^{+} \mathrm{e}^{-}$, $\mu^{+} \mu^{-}, \tau^{+} \tau^{-}$or $\mathrm{q} \overline{\mathrm{q}}$, and JETSET [17] for $\mathrm{e}^{+} \mathrm{e}^{-} \rightarrow$ $\mathrm{q} \overline{\mathrm{q}}(\gamma)$. The Monte Carlo events are passed through a full detector simulation, based on the GEANT program [18], which takes into account the effects of energy loss, multiple scattering, showering and time dependent detector inefficiencies. These events are reconstructed with the same program used for the data. The number of Monte Carlo events in each process is about ten times larger than the corresponding data sample.

\section{Measurement technique}

To measure the tau leptonic branching fractions, first a sample of $\mathrm{e}^{+} \mathrm{e}^{-} \rightarrow \tau^{+} \tau^{-}(\gamma)$ events is selected with some remnant background mainly from other leptonic $\mathrm{Z}$ decays. From this sample the branching fraction of the tau into a lepton $\ell$ is then obtained as:

$\mathcal{B}\left(\tau \rightarrow \ell \bar{v}_{\ell} \nu_{\tau}\right)=\frac{N_{\ell}\left(1-f_{\ell}^{\text {non } \tau}-f_{h \rightarrow l}^{\tau}\right)}{N_{\tau}\left(1-f_{\tau}^{\text {non } \tau}\right)} \frac{1}{\varepsilon_{\ell}^{\mathrm{ID}}} \frac{\varepsilon_{\tau}^{\mathrm{sel}}}{\varepsilon_{\ell}^{\mathrm{sel}}}$,

where $N_{\tau}$ is the number of selected tau decays, $\varepsilon_{\tau}^{\text {sel }}$ is the $\mathrm{e}^{+} \mathrm{e}^{-} \rightarrow \tau^{+} \tau^{-}(\gamma)$ selection efficiency, $f_{\tau}^{\text {non } \tau}$ is the background of other final states, $\varepsilon_{\ell}^{\text {sel }}$ is the selection efficiency for tau decays into the lepton $\ell$, $N_{\ell}$ the number of identified leptons and $\varepsilon_{\ell}^{\mathrm{ID}}$ the identification efficiency of the lepton $\ell$. The quantities

\footnotetext{
9 In this Letter figures and tables often refer just to the 1994 data, which is the largest sample.
}

$f_{h \rightarrow l}^{\tau}$ and $f_{\ell}^{\text {non } \tau}$ are the background contaminations from other $\tau$ decays and non- $\tau$ final states in the selected leptonic decays, respectively.

Systematic uncertainties from the selection efficien$\operatorname{cies} \varepsilon_{\tau}^{\text {sel }}$ and $\varepsilon_{\ell}^{\text {sel }}$ cancel if the ratio $\varepsilon_{\tau}^{\text {sel }} / \varepsilon_{\ell}^{\text {sel }}$ equals unity, i.e., the selection of $\mathrm{e}^{+} \mathrm{e}^{-} \rightarrow \tau^{+} \tau^{-}(\gamma)$ does not introduce a bias to the fractions of leptonic tau decays. This bias is avoided by subdividing each event into two hemispheres by a plane perpendicular to the thrust axis. Then, the selection of $\mathrm{e}^{+} \mathrm{e}^{-} \rightarrow \tau^{+} \tau^{-}(\gamma)$ events is performed using the information from just one hemisphere, called the tagged hemisphere. This information is not correlated with the opposite hemisphere, denoted as the analysis hemisphere, that is subject to electron and muon identification. The $\tau$ decays identified in the analysis hemisphere constitute a bias free sample for the branching fraction measurement.

\section{Selection of $\mathrm{e}^{+} \mathrm{e}^{-} \rightarrow \tau^{+} \tau^{-}(\gamma)$ events}

Events of the process $\mathrm{e}^{+} \mathrm{e}^{-} \rightarrow \tau^{+} \tau^{-}(\gamma)$ are characterised by two low multiplicity jets which are almost back-to-back. To ensure good track measurements in the central tracker, the fiducial volume is defined by $|\cos \theta|<0.72$, where $\theta$ is the polar angle of the thrust axis with respect to the electron beam direction. The requirements for the preselection of leptonic $\mathrm{Z}$ decays are:

- the number of tracks must be less than 10 ,

- the number of tracks in each hemisphere must be less than 7 ,

- each hemisphere must have at least one track with a transverse momentum greater than $1 \mathrm{GeV}$,

- the distance of closest approach of at least one track to the beam position in the plane perpendicular to the beam axis, must be less than $5 \mathrm{~mm}$.

The only additional requirement on the event as a whole is that the acollinearity angle between the leading tracks of the two hemispheres must be larger than $2.8 \mathrm{rad}$. The background suppression due to this cut is illustrated in Fig. 1 for 1994 data after the preselection.

The criteria to select $\mathrm{e}^{+} \mathrm{e}^{-} \rightarrow \tau^{+} \tau^{-}(\gamma)$ events in the tagged hemisphere are:

- there must be one, two or three tracks in the central tracker, 


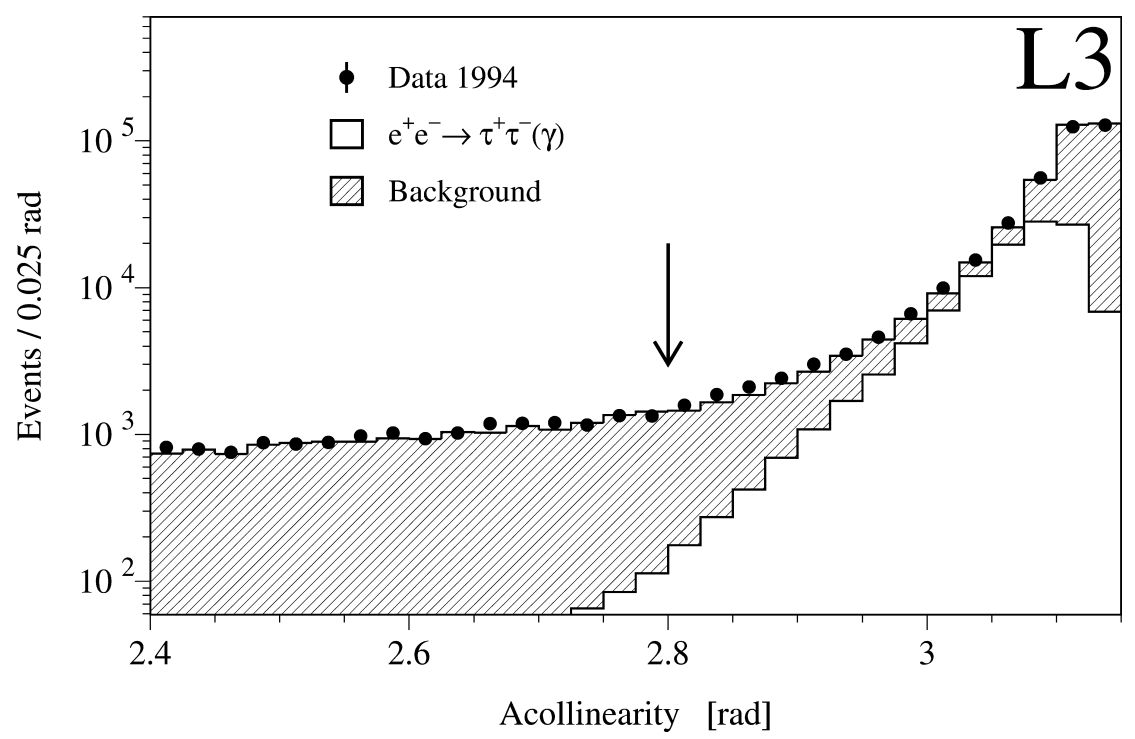

Fig. 1. Distribution of the acollinearity after the preselection. The arrow indicates the position of the cut applied to select $\mathrm{e}^{+} \mathrm{e}^{-} \rightarrow \tau^{+} \tau^{-}(\gamma)$ events.

- the angle between each track and the thrust axis must be less than $0.45 \mathrm{rad}$,

- the energy of the most energetic cluster in the electromagnetic calorimeter, BGO, must be less than $75 \%$ of the beam energy,

- the momentum of a track reconstructed in the muon chambers must be less than $65 \%$ of the beam energy.

Background events that fall into less efficient regions of the detector can fake $\mathrm{e}^{+} \mathrm{e}^{-} \rightarrow \tau^{+} \tau^{-}(\gamma)$ events. They are identified by the following criteria in the tagged hemisphere:

- $\mathrm{e}^{+} \mathrm{e}^{-} \rightarrow \mathrm{e}^{+} \mathrm{e}^{-}(\gamma)$ : there is one track pointing to the carbon fibre support between crystals of the electromagnetic calorimeter, with either its momentum or the corresponding energy measured in the BGO larger than $10 \mathrm{GeV}$. Furthermore, there must be an energy deposit in the hadron calorimeter.

- $\mathrm{e}^{+} \mathrm{e}^{-} \rightarrow \mu^{+} \mu^{-}(\gamma)$ : there is no track in the muon chambers but there is one track in the central tracker with a momentum larger than $15 \mathrm{GeV}$, which points to an energy deposit in the calorimeters compatible with that of a minimum ionising particle.

- $\mathrm{e}^{+} \mathrm{e}^{-} \rightarrow \mathrm{e}^{+} \mathrm{e}^{-} \mathrm{e}^{+} \mathrm{e}^{-}$: there is one track in the central tracker with a momentum of less than $10 \mathrm{GeV}$ pointing to an electromagnetic cluster in the BGO of energy less than $10 \mathrm{GeV}$.

- $\mathrm{e}^{+} \mathrm{e}^{-} \rightarrow \mathrm{e}^{+} \mathrm{e}^{-} \mu^{+} \mu^{-}$: there is no track in the muon chambers but there is one track in the central tracker with a momentum of less than $9 \mathrm{GeV}$ which points to an energy deposit in the calorimeters compatible with the expectation for a minimum ionising particle.

The background candidates passing these cuts are rejected from the $\mathrm{e}^{+} \mathrm{e}^{-} \rightarrow \tau^{+} \tau^{-}(\gamma)$ sample if the acoplanarity of the event is less than 5 mrad. Remaining background from $\mathrm{e}^{+} \mathrm{e}^{-} \rightarrow \mathrm{q} \overline{\mathrm{q}}$ and $\mathrm{e}^{+} \mathrm{e}^{-} \rightarrow$ $\mathrm{e}^{+} \mathrm{e}^{-} \mathrm{q} \overline{\mathrm{q}}$ is suppressed by requiring the energy deposited in the calorimeters to be between 6 and $25 \mathrm{GeV}$ whenever there is more than one track in the tagged hemisphere. Cosmic rays are suppressed by requiring the event time, measured by scintillators, to be within $5 \mathrm{~ns}$ of the beam crossing time for events with a track in the muon chambers or a minimum ionising particle in the calorimeters.

A sample of 163256 tau decays from $\mathrm{e}^{+} \mathrm{e}^{-} \rightarrow$ $\tau^{+} \tau^{-}(\gamma)$ final states is tagged in one hemisphere allowing to study the $\tau$ decay in the analysis hemisphere. The number of tagged hemispheres, $N_{\tau}$, per data taking period is listed in Table 1 . The selection 
Table 1

Number of selected tau decays, number of identified electrons and muons, lepton identification efficiencies, fraction of background from non- $\tau$ final states and other $\tau$ decays for the different data taking periods

\begin{tabular}{ccccc}
\hline & $1991+1992$ & 1993 & 1994 & 26158 \\
\hline$N_{\tau}$ & 42086 & 29620 & 65392 & 3878 \\
$N_{e}\left(\tau \rightarrow \mathrm{e} \bar{\nu}_{\mathrm{e}} \nu_{\tau}\right)$ & 6519 & 4525 & 9754 & $82.75 \pm 0.26$ \\
$\varepsilon_{e}^{\mathrm{ID}}(\%)$ & $85.94 \pm 0.26$ & $83.62 \pm 0.29$ & $83.86 \pm 0.17$ & $2.11 \pm 0.34$ \\
$f_{e}^{\text {non } \tau(\%)}$ & $1.43 \pm 0.23$ & $2.42 \pm 0.38$ & $1.32 \pm 0.19$ & $1.76 \pm 0.10$ \\
$f_{e}^{\tau \rightarrow e}(\%)$ & $2.04 \pm 0.12$ & $1.74 \pm 0.11$ & $1.70 \pm 0.10$ & 3289 \\
$N_{\mu}\left(\tau \rightarrow \mu \bar{v}_{\mu} \nu_{\tau}\right)$ & 5682 & 3984 & 8554 & $75.54 \pm 0.24$ \\
$\varepsilon_{\mu}^{\mathrm{ID}}(\%)$ & $76.22 \pm 0.23$ & $77.65 \pm 0.24$ & $75.10 \pm 0.17$ & $1.60 \pm 0.15$ \\
$f_{\mu}^{\text {non } \tau}(\%)$ & $1.29 \pm 0.16$ & $1.89 \pm 0.23$ & $1.11 \pm 0.09$ & $1.17 \pm 0.08$ \\
$f_{\mu}^{\tau \rightarrow \mu}(\%)$ & $1.41 \pm 0.09$ & $1.38 \pm 0.09$ & $1.44 \pm 0.09$ & \\
\hline
\end{tabular}

efficiency is estimated from Monte Carlo simulation to be $76 \%$.

The remaining background is determined from the data for each year separately. It is estimated by comparing reference distributions in data and Monte Carlo.

The fraction of $\mathrm{e}^{+} \mathrm{e}^{-} \rightarrow \mathrm{e}^{+} \mathrm{e}^{-}(\gamma)$ background is determined using the energy distribution measured in the electromagnetic calorimeter in the analysis hemisphere, as shown in Fig. 2a for the 1994 data sample. The high end of this spectrum is dominated by $\mathrm{e}^{+} \mathrm{e}^{-} \rightarrow \mathrm{e}^{+} \mathrm{e}^{-}(\gamma)$ events with a small contribution from $\mathrm{e}^{+} \mathrm{e}^{-} \rightarrow \tau^{+} \tau^{-}(\gamma)$ events. A fit is performed to the data distribution, in which the shapes of the $\mathrm{e}^{+} \mathrm{e}^{-} \rightarrow \mathrm{e}^{+} \mathrm{e}^{-}(\gamma)$ and $\mathrm{e}^{+} \mathrm{e}^{-} \rightarrow \tau^{+} \tau^{-}(\gamma)$ contributions are taken from Monte Carlo and the normalisation of the $\mathrm{e}^{+} \mathrm{e}^{-} \rightarrow \mathrm{e}^{+} \mathrm{e}^{-}(\gamma)$ background is a free parameter.

A similar procedure is used for the other background sources. The fraction of background from $\mathrm{e}^{+} \mathrm{e}^{-} \rightarrow \mu^{+} \mu^{-}(\gamma)$ events is estimated using the muon momentum distribution measured in the analysis hemisphere, as displayed for the 1994 data sample in Fig. 2b. The contamination from two-photon processes is estimated using the acollinearity distribution and the fraction of $\mathrm{e}^{+} \mathrm{e}^{-} \rightarrow \mathrm{q} \overline{\mathrm{q}}$ background by means of the distribution of the total energy deposited in the calorimeters. The contamination from cosmic rays is estimated from data only, using the sidebands of the two-dimensional distribution of the distance of closest approach from the two leading tracks in each hemisphere.

The approximate contributions to the $\mathrm{e}^{+} \mathrm{e}^{-} \rightarrow$ $\tau^{+} \tau^{-}(\gamma)$ sample from the different background sources are: $1.2 \%$ from $\mathrm{e}^{+} \mathrm{e}^{-} \rightarrow \mathrm{e}^{+} \mathrm{e}^{-}(\gamma), 1 \%$ from $\mathrm{e}^{+} \mathrm{e}^{-} \rightarrow \mu^{+} \mu^{-}(\gamma), 0.25 \%$ from $\mathrm{e}^{+} \mathrm{e}^{-} \rightarrow \mathrm{q} \overline{\mathrm{q}}, 0.3 \%$ from two-photon events and $0.1 \%$ from cosmic rays. The detailed summary of the estimated background fractions is given in Table 2.

\section{Lepton identification}

The lepton identification [19] is performed in the analysis hemisphere combining the informations from several subdetectors. In particular, the electromagnetic calorimeter is essential for the identification of electrons, while the muon chambers and the hadron calorimeter allow for the identification of muons. Pions are a potential source of contamination both for electrons and muons. The $\rho$ mesons can be misidentified as electrons when the showers from the charged and neutral pions overlap.

Electrons are characterised by a track in the central tracker pointing to an energy deposit in the BGO, 

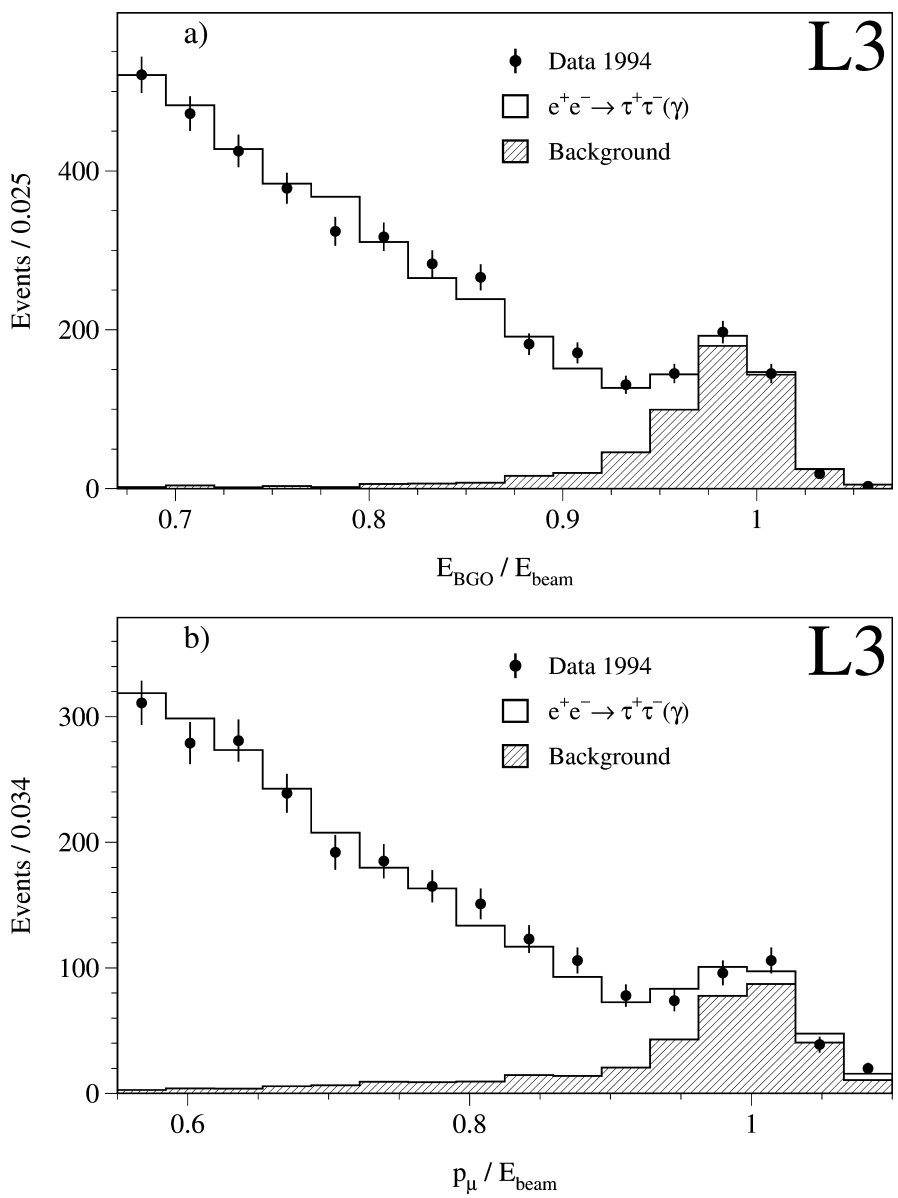

Fig. 2. Distribution of (a) the energy in the electromagnetic calorimeter, BGO, and (b) the muon momentum. Both quantities are normalised to the beam energy, $E_{\text {beam }}$, and measured in the analysis hemisphere of events tagged as $\mathrm{e}^{+} \mathrm{e}^{-} \rightarrow \tau^{+} \tau^{-}(\gamma)$.

Table 2

Summary of the background fractions in the $\mathrm{e}^{+} \mathrm{e}^{-} \rightarrow \tau^{+} \tau^{-}(\gamma)$ sample

\begin{tabular}{|c|c|c|c|c|}
\hline \multirow[t]{2}{*}{ Channel } & \multicolumn{4}{|c|}{ Background in \% } \\
\hline & $1991+1992$ & 1993 & 1994 & 1995 \\
\hline $\mathrm{e}^{+} \mathrm{e}^{-} \rightarrow \mu^{+} \mu^{-}(\gamma)$ & $0.96 \pm 0.08$ & $0.97 \pm 0.09$ & $0.94 \pm 0.05$ & $1.11 \pm 0.08$ \\
\hline $\mathrm{e}^{+} \mathrm{e}^{-} \rightarrow \mathrm{e}^{+} \mathrm{e}^{-}(\gamma)$ & $1.19 \pm 0.14$ & $1.67 \pm 0.19$ & $1.16 \pm 0.07$ & $1.33 \pm 0.11$ \\
\hline $\mathrm{e}^{+} \mathrm{e}^{-} \rightarrow \mathrm{e}^{+} \mathrm{e}^{-} \mu^{+} \mu^{-}$ & $0.03 \pm 0.01$ & $0.12 \pm 0.02$ & $0.03 \pm 0.01$ & $0.14 \pm 0.02$ \\
\hline $\mathrm{e}^{+} \mathrm{e}^{-} \rightarrow \mathrm{e}^{+} \mathrm{e}^{-} \mathrm{e}^{+} \mathrm{e}^{-}$ & $0.15 \pm 0.03$ & $0.27 \pm 0.04$ & $0.15 \pm 0.02$ & $0.29 \pm 0.05$ \\
\hline $\mathrm{e}^{+} \mathrm{e}^{-} \rightarrow \mathrm{e}^{+} \mathrm{e}^{-} \mathrm{q} \overline{\mathrm{q}}$ & $0.04 \pm 0.02$ & $0.06 \pm 0.03$ & $0.07 \pm 0.02$ & $0.06 \pm 0.03$ \\
\hline Cosmic rays & $0.04 \pm 0.01$ & $0.11 \pm 0.02$ & $0.10 \pm 0.01$ & $0.17 \pm 0.03$ \\
\hline $\mathrm{e}^{+} \mathrm{e}^{-} \rightarrow \mathrm{q} \overline{\mathrm{q}}$ & $0.22 \pm 0.05$ & $0.23 \pm 0.06$ & $0.24 \pm 0.05$ & $0.14 \pm 0.03$ \\
\hline Total & $2.64 \pm 0.17$ & $3.42 \pm 0.23$ & $2.70 \pm 0.10$ & $3.24 \pm 0.15$ \\
\hline
\end{tabular}



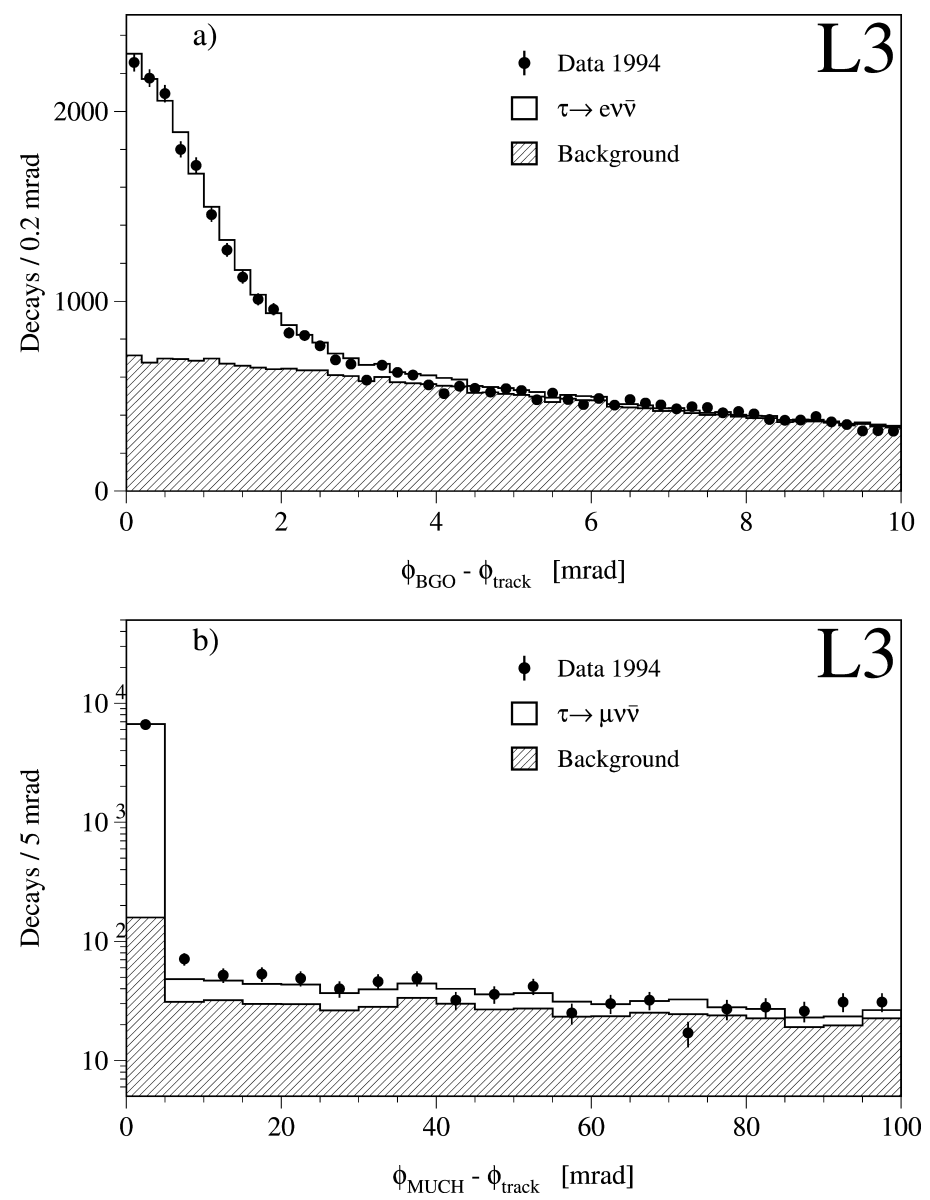

Fig. 3. Distribution of the difference of the azimuthal angles measured by (a) the central tracker and the electromagnetic calorimeter (BGO) for electron candidates and (b) the central tracker and the muon chambers (MUCH) for muon candidates. The background contributions are shown as the hatched histograms.

that must be of electromagnetic shape. The distribution of the difference of the azimuthal angles $\phi$ measured by the central tracker and the electromagnetic calorimeter is shown in Fig. 3a. The matching requirement depends on the electromagnetic energy and varies in $\phi$ between 10 and $3 \mathrm{mrad}$ in the energy range from 2 to $45 \mathrm{GeV}$. Similar cuts are applied to the difference in the polar angle. In addition, the energy measurement of the BGO must be compatible with the track momentum. This criterion and the angular matching are relaxed for tracks in the anode wire region of the drift chamber and the requirements on the electromagnetic shower shape are tightened. Energy deposits in the hadron calorimeter must be consistent with the tail of an electromagnetic shower.

Muons are identified as a track in the muon chambers matching with a track in the central tracker originating from the interaction region. Furthermore, the energy deposit in the calorimeters must correspond to the expectations for a minimum ionising particle. The distribution of the difference in the angle $\phi$ measured in the central tracker and the muon chambers is shown in Fig. 3b. Muon tracks originating from $\tau$ decays are well separated from hadron punch-through. Muons reaching the muon chambers lose energy in 
the calorimeters, resulting in a momentum threshold of about $2.5 \mathrm{GeV}$.

\section{Efficiencies and background estimation}

The efficiencies of the lepton identification estimated from Monte Carlo are smooth functions of the lepton energy and their average value is about $90 \%$ for electrons and $75 \%$ for muons. These efficiencies are corrected using data samples enriched in $\mathrm{e}^{+} \mathrm{e}^{-} \rightarrow$ $\mathrm{e}^{+} \mathrm{e}^{-}(\gamma), \mathrm{e}^{+} \mathrm{e}^{-} \rightarrow \mu^{+} \mu^{-}(\gamma), \mathrm{e}^{+} \mathrm{e}^{-} \rightarrow \mathrm{e}^{+} \mathrm{e}^{-} \mathrm{e}^{+} \mathrm{e}^{-}$ and $\mathrm{e}^{+} \mathrm{e}^{-} \rightarrow \mathrm{e}^{+} \mathrm{e}^{-} \mu^{+} \mu^{-}$events, selected by identifying a lepton in the tagged hemisphere.

The statistics available from $\mathrm{e}^{+} \mathrm{e}^{-} \rightarrow \mathrm{e}^{+} \mathrm{e}^{-} \mathrm{e}^{+} \mathrm{e}^{-}$ and $\mathrm{e}^{+} \mathrm{e}^{-} \rightarrow \mathrm{e}^{+} \mathrm{e}^{-} \mu^{+} \mu^{-}$events is large at low lepton energy, while that from $\mathrm{e}^{+} \mathrm{e}^{-} \rightarrow \mathrm{e}^{+} \mathrm{e}^{-}(\gamma)$ and $\mathrm{e}^{+} \mathrm{e}^{-} \rightarrow \mu^{+} \mu^{-}(\gamma)$ events is large at high lepton energy. Fig. 4 displays the ratio of the identification efficiencies obtained from data and Monte Carlo, together with the result of a linear fit, for electrons and muons, respectively. The result of the fit is applied as an energy dependent scale factor to the corresponding identification efficiencies determined from the Monte Carlo. These scale factors, obtained for each year separately, are near unity and almost constant over the full energy range.

The background in the lepton sample from $\mathrm{e}^{+} \mathrm{e}^{-} \rightarrow$ $\mathrm{e}^{+} \mathrm{e}^{-}(\gamma)$ and $\mathrm{e}^{+} \mathrm{e}^{-} \rightarrow \mu^{+} \mu^{-}(\gamma)$ is estimated using the same procedure as for the $\mathrm{e}^{+} \mathrm{e}^{-} \rightarrow \tau^{+} \tau^{-}(\gamma)$ selection. The fraction of hadronic tau decays which passes the lepton identification is determined from Monte Carlo simulation.

\section{Systematic uncertainties}

Systematic uncertainties result from the preselection, the cut on the acollinearity angle, the selection of $\mathrm{e}^{+} \mathrm{e}^{-} \rightarrow \tau^{+} \tau^{-}(\gamma)$ events, the background estimations, the lepton identification efficiency and the range of the lepton energy used in the measurement.

The uncertainties from the preselection, the cut on the acollinearity and the hemisphere tagging criteria are estimated by varying the corresponding requirements inside reasonable ranges. The change in the branching fraction is assigned as systematic uncertainty. The background from $\mathrm{e}^{+} \mathrm{e}^{-} \rightarrow \mathrm{e}^{+} \mathrm{e}^{-}(\gamma)$,
Table 3

Uncorrelated systematic uncertainties for the 1994 data sample and their combination

\begin{tabular}{lcc}
\hline Source & $\mathcal{B}\left(\tau \rightarrow \mathrm{e} \bar{v}_{\mathrm{e}} \nu_{\tau}\right)$ & $\mathcal{B}\left(\tau \rightarrow \mu \bar{\nu}_{\mu} \nu_{\tau}\right)$ \\
\hline Preselection and acollinearity angle & 0.040 & 0.031 \\
Selection of $\mathrm{e}^{+} \mathrm{e}^{-} \rightarrow \tau^{+} \tau^{-}(\gamma)$ & 0.026 & 0.029 \\
Uncorrelated background & 0.031 & 0.021 \\
Lepton identification scale factor & 0.041 & 0.048 \\
Lepton energy range & 0.054 & 0.028 \\
Monte Carlo statistics & 0.053 & 0.057 \\
Total uncertainty & 0.103 & 0.093 \\
\hline
\end{tabular}

$\mathrm{e}^{+} \mathrm{e}^{-} \rightarrow \mu^{+} \mu^{-}(\gamma)$ and two-photon processes is obtained from fits to the data. The statistical error of these fits is taken as the systematic uncertainty. The systematic uncertainty from the efficiency is obtained from the statistical errors of the energy dependent scale factors. The Monte Carlo statistical uncertainty is also considered.

These uncertainties are estimated for each year separately [19], as an example, the uncertainties for the 1994 data are given in Table 3. They are considered as uncorrelated and their combined values for the full data set are given in Table 4. This table also presents the systematic uncertainties fully correlated between the data sets of the different years. These result from the background shapes used in the fit of the $\mathrm{e}^{+} \mathrm{e}^{-} \rightarrow \mathrm{e}^{+} \mathrm{e}^{-}(\gamma), \mathrm{e}^{+} \mathrm{e}^{-} \rightarrow \mu^{+} \mu^{-}(\gamma)$ and two-photon backgrounds and the uncertainty on the fraction of misidentified hadrons. The uncertainty of the one-prong branching fraction of the $\tau$ into hadrons and the $\tau$ polarisation are also treated as correlated. Their effect is estimated by varying them within their uncertainties [20,21] and quoting the change of the leptonic branching fraction.

Table 5 lists the sources of systematic uncertainties correlated between $\mathcal{B}\left(\tau \rightarrow \mathrm{e} \bar{v}_{\mathrm{e}} \nu_{\tau}\right)$ and $\mathcal{B}\left(\tau \rightarrow \mu \bar{v}_{\mu} v_{\tau}\right)$. They are taken into account to derive $\mathcal{B}\left(\tau \rightarrow \ell \bar{v}_{\ell} \nu_{\tau}\right)$ and $g_{\mu} / g_{\mathrm{e}}$.

\section{Results}

Fig. 5 displays the spectra of electrons and muons identified in the analysis hemisphere in the full data 

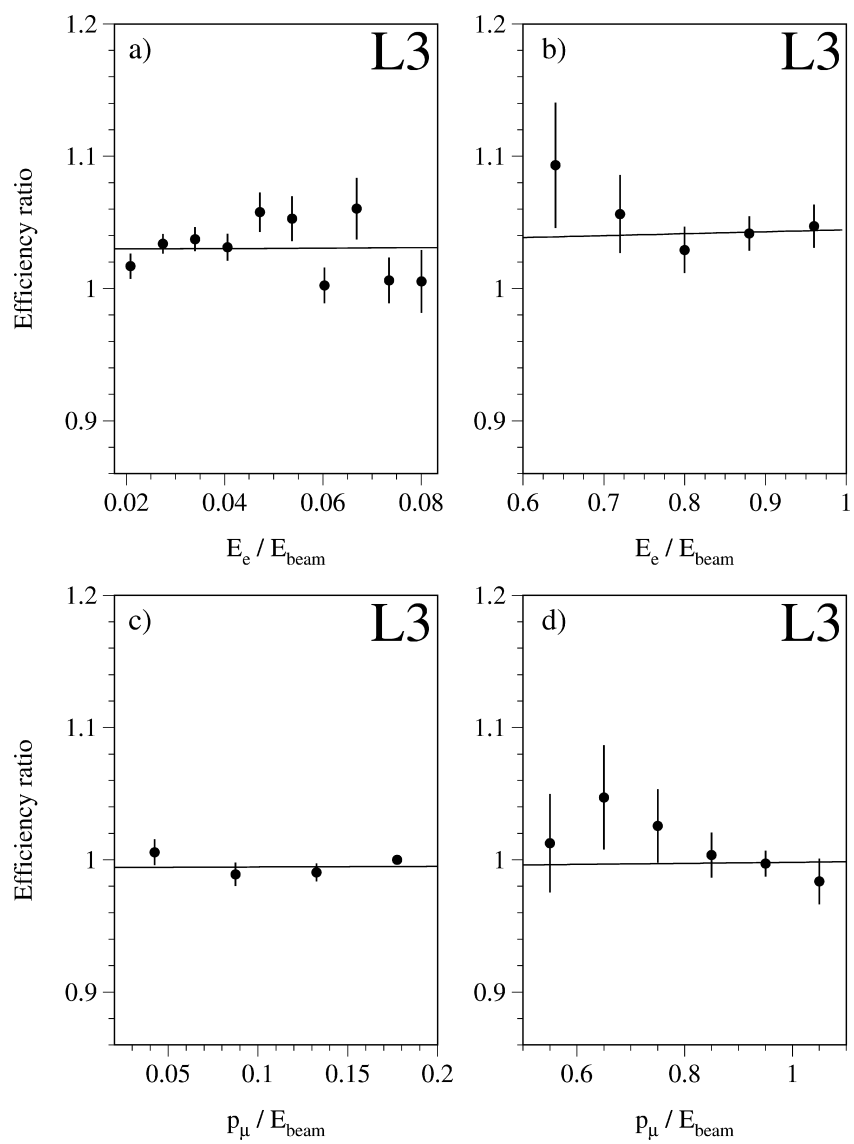

Fig. 4. Ratios of the Monte Carlo to data identification efficiencies for electrons and muons as a function of the normalised electron energy and muon momentum. They are obtained for (a) $\mathrm{e}^{+} \mathrm{e}^{-} \rightarrow \mathrm{e}^{+} \mathrm{e}^{-} \mathrm{e}^{+} \mathrm{e}^{-}$, (b) $\mathrm{e}^{+} \mathrm{e}^{-} \rightarrow \mathrm{e}^{+} \mathrm{e}^{-}(\gamma)$, (c) $\mathrm{e}^{+} \mathrm{e}^{-} \rightarrow \mathrm{e}^{+} \mathrm{e}^{-} \mu^{+} \mu^{-}$and (d) $\mathrm{e}^{+} \mathrm{e}^{-} \rightarrow \mu^{+} \mu^{-}(\gamma)$ events, for the 1994 data. The straight line is the result of a linear fit over the full energy range.

Table 4

Correlated and uncorrelated systematic uncertainties for the full data sample

\begin{tabular}{lcc}
\hline Source & $\mathcal{B}\left(\tau \rightarrow \mathrm{e} \overline{\mathrm{e}}_{\mathrm{e}} \nu_{\tau}\right)$ & $\mathcal{B}\left(\tau \rightarrow \mu \bar{\nu}_{\mu} \nu_{\tau}\right)$ \\
\hline Correlated background & 0.036 & 0.025 \\
Hadronic 1-prong branching & & \\
$\quad$ fraction & 0.021 & 0.018 \\
Polarisation & 0.003 & 0.003 \\
Total correlated uncertainty & 0.042 & 0.031 \\
Total uncorrelated uncertainty & 0.063 & 0.059 \\
Total uncertainty & 0.076 & 0.067 \\
\hline
\end{tabular}

Table 5

Systematic uncertainties on $\mathcal{B}\left(\tau \rightarrow \ell \bar{v}_{\ell} \nu_{\tau}\right)$ treated as fully correlated for the electron and muon channels

\begin{tabular}{lc}
\hline Source & $\mathcal{B}\left(\tau \rightarrow \ell \bar{v}_{\ell} \nu_{\tau}\right)$ \\
\hline Background in the $\tau$ sample & 0.013 \\
Hadronic 1-prong branching fraction & 0.013 \\
Polarisation & 0.003 \\
Acollinearity angle & 0.012 \\
Selection & 0.004 \\
Total uncertainty & 0.023 \\
\hline
\end{tabular}



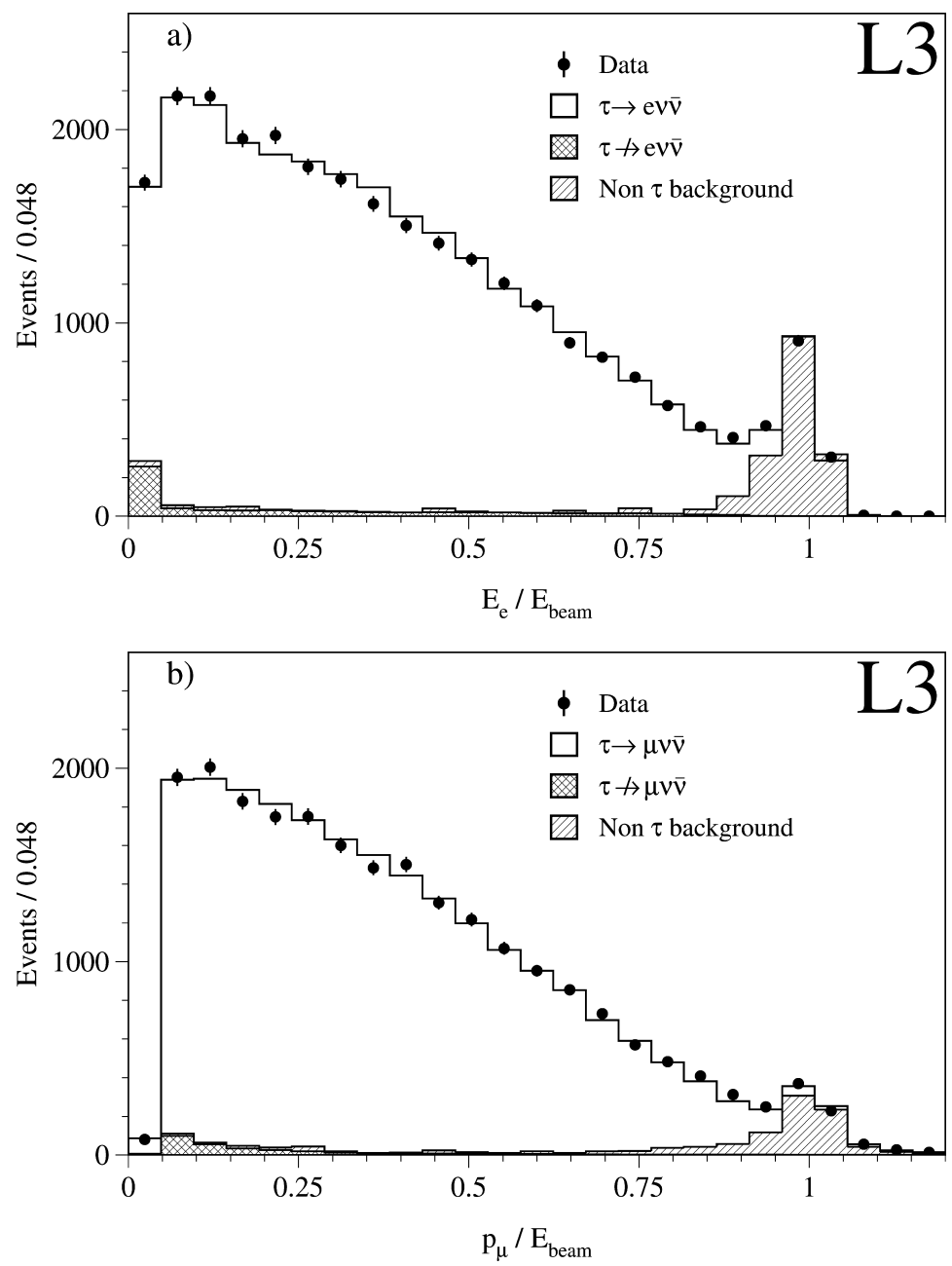

Fig. 5. The spectra of (a) electrons and (b) muons from tau decays in the full data sample. The expectations from the Monte Carlo simulation and the background from other tau decays and other leptonic final states are also shown.

sample. The spectra obtained from the Monte Carlo simulations of $\tau \rightarrow \mathrm{e} \bar{\nu}_{\mathrm{e}} \nu_{\tau}$ and $\tau \rightarrow \mu \bar{v}_{\mu} \nu_{\tau}$, corrected for the identification efficiency scale factor and the background sources are also shown. The branching ratios are determined using leptons with energies normalised to the beam energy that range from 0.02 to 0.85 for electrons and from 0.05 to 0.92 for muons. In these ranges the efficiencies are almost flat and the background is small. The number of events inside these ranges, the lepton identification efficiencies and the background fractions are given in Table 1. Taking these numbers and using Eq. (4), the branching fractions of the tau lepton into electron and muon are:

$\mathcal{B}\left(\tau \rightarrow \mathrm{e} \bar{\nu}_{\mathrm{e}} v_{\tau}\right)=(17.806 \pm 0.104 \pm 0.076) \%$

and

$\mathcal{B}\left(\tau \rightarrow \mu \bar{v}_{\mu} \nu_{\tau}\right)=(17.342 \pm 0.110 \pm 0.067) \%$,

where the first uncertainty is statistical and the second systematic. These values are in good agreement with the current world average [20]. The results are used to test lepton universality for the charged weak currents. The ratio of the charged current coupling constants of 
the muon and the electron, is obtained as:

$g_{\mu} / g_{\mathrm{e}}=1.0007 \pm 0.0043 \pm 0.0027$,

supporting the lepton universality hypothesis. Assuming electron-muon universality, the branching fraction of the tau into leptons is:

$\mathcal{B}\left(\tau \rightarrow \ell \bar{v}_{\ell} \nu_{\tau}\right)=(17.818 \pm 0.077 \pm 0.053) \%$.

Together with our measurement of the tau lifetime [22] the Fermi constant in tau lepton decays is determined as:

$G_{F}=(1.1616 \pm 0.0058) \times 10^{-5} \mathrm{GeV}^{-2}$.

Furthermore, from the branching fraction of the tau into leptons, $R_{\tau}$ is obtained using Eq. (2) as:

$R_{\tau}=3.640 \pm 0.030$.

From Eq. (3), the value of the strong coupling constant at the tau mass is:

$\alpha_{s}\left(m_{\tau}^{2}\right)=0.322 \pm 0.009$ (exp.) \pm 0.015 (theory).

The first error is due to the uncertainties of the tau leptonic branching fraction and the CKM matrix elements [20]. The second error is the quadratic sum of the uncertainties resulting from the renormalisation scale, the fourth order term in $\alpha_{s}$, the electroweak corrections $S_{\mathrm{EW}}$ and the the non-perturbative correction $\delta_{\mathrm{NP}}$. The dominant contribution to the error is the renormalisation scale uncertainty, which is estimated following Ref. [23]. Other theoretical uncertainties discussed in Ref. [24] are not considered. The value of $\alpha_{s}\left(m_{\tau}^{2}\right)$ is extrapolated to the $\mathrm{Z}$ mass scale using the renormalisation group equation [25] with the four loop calculation of the QCD $\beta$-functions [26]. The result,

$\alpha_{s}\left(m_{\mathrm{Z}}^{2}\right)=0.120 \pm 0.002$,

is in good agreement with the value obtained by L3 from the study of hadronic events at the $Z$ peak [27] and the current world average [20].

\section{Acknowledgements}

We thank A. Kataev for discussions about the estimation of the theoretical uncertainty of $R_{\tau}$. We wish to express our gratitude to the CERN accelerator divisions for the excellent performance of the LEP machine. We acknowledge the contributions of the engineers and technicians who have participated in the construction and maintenance of this experiment.

\section{References}

[1] S.L. Glashow, Nucl. Phys. 22 (1961) 579; S. Weinberg, Phys. Rev. Lett. 19 (1967) 1264;

A. Salam, in: N. Svartholm (Ed.), Elementary Particle Theory, Almqvist \& Wiksell, Stockholm, 1968, p. 367.

[2] W.J. Marciano, A. Sirlin, Phys. Rev. Lett. 61 (1988) 1815.

[3] T. Kinoshita, A. Sirlin, Phys. Rev. 113 (1959) 1652.

[4] S.M. Berman, A. Sirlin, Ann. Phys. 20 (1962) 20.

[5] W.J. Marciano, Nucl. Phys. Proc. Suppl. 40 (1995) 3; T. Riemann, private communication.

[6] S.G. Gorishny, A.L. Kataev, S.A. Larin, Phys. Lett. B 259 (1991) 144.

[7] A.L. Kataev, V.V. Starshenko, Mod. Phys. Lett. A 10 (1995) 235.

[8] N. Cabibbo, Phys. Rev. Lett. 10 (1963) 531; M. Kobayashi, T. Maskawa, Prog. Theor. Phys. 49 (1973) 652.

[9] A. Sirlin, Nucl. Phys. B 196 (1982) 83.

[10] M. Neubert, Nucl. Phys. B 463 (1996) 511.

[11] L3 Collaboration, B. Adeva et al., Nucl. Instrum. Methods A 289 (1990) 35;

J.A. Bakken et al., Nucl. Instrum. Methods A 275 (1989) 81;

O. Adriani et al., Nucl. Instrum. Methods A 302 (1991) 53;

B. Adeva et al., Nucl. Instrum. Methods A 323 (1992) 109;

K. Deiters et al., Nucl. Instrum. Methods A 323 (1992) 162;

M. Chemarin et al., Nucl. Instrum. Methods A 349 (1994) 345; M. Acciarri et al., Nucl. Instrum. Methods A 351 (1994) 300;

G. Basti et al., Nucl. Instrum. Methods A 374 (1996) 293;

A. Adam et al., Nucl. Instrum. Methods A 383 (1996) 342.

[12] L3 Collaboration, O. Adriani et al., Phys. Rep. 236 (1993) 1.

[13] OPAL Collaboration, G. Abbiendi et al., Phys. Lett. B 447 (1999) 134;

DELPHI Collaboration, P. Abreu et al., Eur. Phys. J. C 10 (1999) 201;

CLEO Collaboration, A. Anastassow et al., Phys. Rev. D 55 (1997) 2559;

ALEPH Collaboration, D. Buskulic et al., Z. Phys. C 70 (1996) 561 ;

OPAL Collaboration, R. Akers et al., Z. Phys. C 66 (1995) 543; ARGUS Collaboration, H. Albrecht et al., Phys. Lett. B 316 (1993) 608;

ALEPH Collaboration, D. Decamp et al., Z. Phys. C 54 (1992) 211.

[14] S. Jadach, B.F.L. Ward, Z. Wạs, Comp. Phys. Commun. 79 (1994) 503

[15] J.H. Field, Phys. Lett. B 323 (1994) 432; J.H. Field, T. Riemann, Comp. Phys. Commun. 94 (1996) 53.

[16] F.A. Berends, P.H. Daverfeldt, R. Kleiss, Nucl. Phys. B 253 (1985) 441.

[17] T. Sjöstrand, Comp. Phys. Commun. 39 (1986) 347; 
T. Sjöstrand, M. Bengtsson, Comp. Phys. Commun. 43 (1987) 367.

[18] R. Brun et al., Preprint CERN DD/EE/84-1 (1984), revised September 1987. The GHEISHA program (H. Fesefeldt, RWTH Aachen Report PITHA 85/02, 1985) is used to simulate hadronic interactions.

[19] F. Ziegler, Ph.D. Thesis, Humboldt University, Berlin, 2000.

[20] D.E. Groom et al., Eur. Phys. J. C 15 (2000) 1.

[21] L3 Collaboration, M. Acciarri et al., Phys. Lett. B 429 (1998) 387.
[22] L3 Collaboration, M. Acciarri et al., Phys. Lett. B 479 (2000) 67.

[23] F. Le Diberder, A. Pich, Phys. Lett. B 286 (1992) 147.

[24] G. Altarelli, P. Nason, G. Ridolfi, Z. Phys. C 68 (1995) 257.

[25] G. Rodrigo, A. Pich, A. Santamaria, Phys. Lett. B 424 (1998) 367.

[26] T. van Ritbergen, J.A.M. Vermaseren, S.A. Larin, Phys. Lett. B 400 (1997) 379.

[27] L3 Collaboration, M. Acciarri et al., Phys. Lett. B 411 (1997) 339. 